\title{
Corticosteroids and Influenza A associated Acute Respiratory Distress Syndrome
}

\author{
Philippe R. Bauer, MD, PhD \\ Vivek N. lyer, MD, MPH \\ Pulmonary and Critical Care Medicine \\ Mayo Clinic \\ Rochester, MN USA
}

\begin{abstract}
The use of corticosteroids remains controversial in influenza infection, especially with lower respiratory tract infection. We present a case of moderate acute respiratory distress syndrome (ARDS) associated with influenza A that showed a dramatic improvement with combined corticosteroids and antiviral therapy. Host defense against virus infection consists of both innate and adaptive immune responses. An exuberant immune response to the primary pathogen leads to 'collateral' lung damage resulting in ARDS. The use of corticosteroids to modulate this excessive immune response, although intuitive, has been associated with increased mortality when administered early in the course of severe influenza A pneumonia. The administration of corticosteroids in this case was associated with a dramatic and unequivocal improvement. This unique case highlights the potential benefits of corticosteroids use in influenza $A$ associated ARDS and may challenge clinicians to rethink current recommendations that specifically discourage corticosteroids use in patients with Influenza A associated ARDS.
\end{abstract}

\section{Introduction}

The impact of corticosteroids on clinical outcome in patients with influenza $A$ associated respiratory failure is unclear (1). Retrospective studies suggest an adverse effect from early parenteral corticosteroids use in patients with pandemic influenza infection. On the other hand, in immunosuppressed patients, high dose corticosteroid given at the time of diagnosis of influenza was associated with a reduced risk for mechanical ventilation, without increased adverse effects other than delayed viral clearance. In general, the effect of corticosteroids on acute respiratory distress syndrome (ARDS) is controversial and its use is not routinely recommended. The adjunctive use of prednisone during the early phase of community-acquired pneumonia may actually reduce the development of ARDS (2). In severe influenza, early corticosteroids showed no evidence of benefit and suggested potential harm (3). We present a case of moderate ARDS associated with influenza $A$ that showed a dramatic and unequivocal improvement after initiation of corticosteroids.

\section{Abbreviations:}

APACHE: Acute Physiology and Chronic Health Evaluation

ARDS: Acute Respiratory Distress Syndrome

ICU: Intensive Care Unit 
PCR: Polymerase Chain Reaction

SOFA: Sequential Organ Failure Assessment

\section{Case Report}

A 62-year old male, nonsmoker, with a history of hypertension, dyslipidemia and depression, presented in March 2014 with chills, fever and nonproductive cough; he was initially treated for 'bronchitis' as an outpatient with levofloxacin. He had not received the influenza vaccine. Three days later, he developed acute hypoxemic respiratory failure with bilateral pulmonary infiltrates and was hospitalized elsewhere. Influenza testing was negative and he was started on piperacillin/tazobactam and azithromycin. He was transferred to our facility the next day because of worsening respiratory status. Initial heart rate was $80 \mathrm{bpm}$, blood pressure was 120/60 mm Hg, respirations was $22 / \mathrm{min}$, and temperature was $37.7^{\circ} \mathrm{C}$. The Acute Physiology and Chronic Health Evaluation (APACHE) IV score was 55 and the Sequential Organ Failure Assessment (SOFA) score was 8. His presentation was consistent with moderate ARDS with a $\mathrm{PaO}_{2} / \mathrm{FiO}_{2}$ ratio of 143 , a chest radiograph showing bilateral pulmonary infiltrates (Figure 1) and no evidence of heart failure confirmed by bedside echocardiogram.

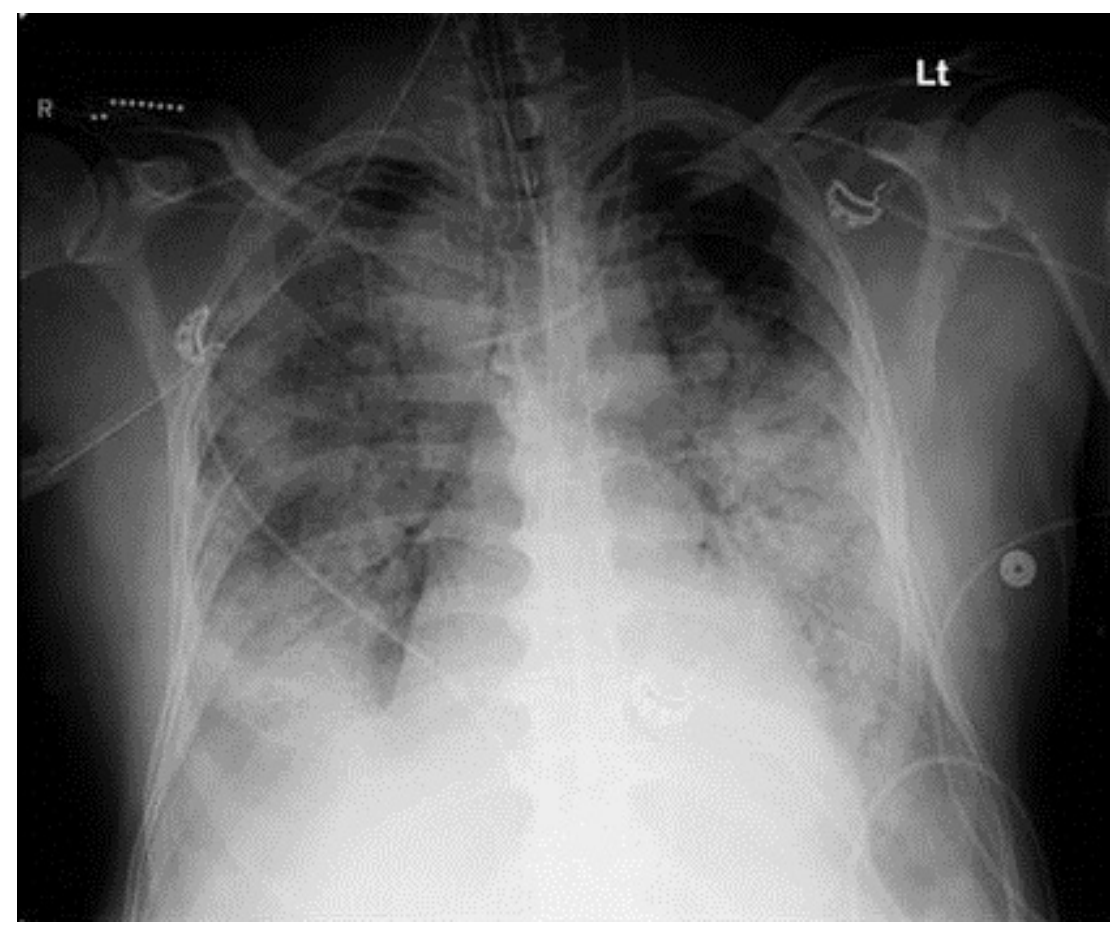

Figure 1. Bilateral pulmonary opacities consistent with moderate ARDS $\left(\mathrm{PaO}_{2} / \mathrm{FiO}_{2}\right.$ ratio 143).

Nasal swab was again negative for influenza by polymerase chain reaction (PCR). Leukocyte count was $4.4 \times 10^{9} / \mathrm{L}$ with lymphopenia $\left(0.22 \times 10^{9} / \mathrm{L}\right)$, hemoglobin was 11.7 $\mathrm{g} / \mathrm{dL}$, and platelet count was $216 \times 10^{9} / \mathrm{L}$. Sodium was $134 \mathrm{mmol} / \mathrm{L}$, creatinine was 1 
$\mathrm{mg} / \mathrm{dL}$ and AST was $142 \mathrm{U} / \mathrm{L}$. He was initiated of high flow nasal oxygen, and vancomycin and oseltamivir were added. Due to the severity of his condition, he was also started on methylprednisolone (125 mg intravenously every 8 hours). After a brief trial of noninvasive ventilation, he was intubated, sedated, paralyzed and placed on a low tidal volume strategy with an initial PEEP of $15 \mathrm{~cm} \mathrm{H} \mathrm{H}_{2} \mathrm{O}$ and a $\mathrm{FiO}_{2}$ of 0.7. A broncho-alveolar lavage, performed post intubation about 16 hours after admission to our facility, showed 35\% alveolar macrophages, $8 \%$ lymphocytes and $57 \%$ neutrophils and was positive for influenza A by PCR; cultures were negative for other organisms. Other tests including HIV, RSV, Mycoplasma, Legionella and urine for Streptococcus antigen were all negative. The patient improved rapidly. He was extubated two days later, and continued on prednisone ( $40 \mathrm{mg}$ daily) for five more days when he was dismissed home without any need for supplemental oxygen, although the chest radiograph continued to show infiltrates.

\section{Discussion}

This case illustrates a patient with delayed diagnosis and treatment of influenza A associated with moderate ARDS who made a rapid and complete recovery with antiviral, antibiotic and adjunctive high dose corticosteroid therapy.

The diagnosis of influenza A in this case meets all criteria established by Clinical Practice Guidelines of the Infectious Diseases Society of America (4). Rapid influenza testing lack sensitivity and false negative are not infrequent. ARDS is a well-defined complication of influenza infection. While the administration of corticosteroids appeared to temporally co-relate with clinical improvement, a causal link cannot be established definitively. The role of immunosuppression in influenza associated ARDS is very controversial with conflicting evidence from prospective (supportive) and retrospective (against) studies. For example, the combined use of sirolimus and prednisone was associated with significantly improved oxygenation as well as reduced organ dysfunction in mechanically ventilated patients with severe H1N1 respiratory failure (5). On the other hand, retrospective studies have shown increased mortality with the early use of high dose corticosteroids in severe influenza A pneumonia and respiratory failure. Furthermore, corticosteroids are now rarely used in ARDS and only sparingly given in case of refractory septic shock. The immune response to influenza infection depends on the virus, the host and the host response to infection. Host defense against virus infection consists of both innate and adaptive immune responses. An excessive immune response may result in 'collateral damage' and critical respiratory illness which may be ameliorated by the use of systemic corticosteroids. On the other hand, suppression of the host immune system may enhance viral replication and prolong critical illness. As a result of these conflicting data, major societies have been unable to firmly recommend for or against corticosteroids therapy in Influenza A associated respiratory failure.

In conclusion, we report on a case of Influenza A with ARDS and rapid improvement on corticosteroids. We have reviewed the current uncertainty surrounding the use of corticosteroids in this setting and leave open the possibility for careful consideration of 
this adjunctive therapy in other cases. Randomized trials are needed to further delineate the potential benefit of corticosteroids in severe influenza infection.

\section{References}

1. Rodrigo C, Leonardi-Bee J, Nguyen-Van-Tam J, Lim WS. Corticosteroids as adjunctive therapy in the treatment of influenza. Cochrane Database Syst Rev. 2016 Mar 7;3:CD010406. [CrossRef] [PubMed]

2. Blum CA, Nigro N, Briel M, et al. Adjunct prednisone therapy for patients with community-acquired pneumonia: a multicentre, double-blind, randomised, placebocontrolled trial. Lancet. 2015 Apr 18;385(9977):1511-8. [CrossRef] [PubMed]

3. Brun-Buisson C, Richard JC, Mercat A, Thiébaut AC, Brochard L; REVA-SRLF A/H1N1v 2009 Registry Group. Early corticosteroids in severe influenza A/H1N1 pneumonia and acute respiratory distress syndrome. Am J Respir Crit Care Med. 2011 May 1;183(9):1200-6. [CrossRef] [PubMed]

4. Harper SA, Bradley JS, Englund JA, et al. Seasonal influenza in adults and children-diagnosis, treatment, chemoprophylaxis, and institutional outbreak management: clinical practice guidelines of the Infectious Diseases Society of America. Clin Infect Dis. 2009 Apr 15;48(8):1003-32. [CrossRef] [PubMed]

5. Wang $\mathrm{CH}$, Chung FT, Lin SM, Huang SY, Chou CL, Lee KY, Lin TY, Kuo HP. Adjuvant treatment with a mammalian target of rapamycin inhibitor, sirolimus, and steroids improves outcomes in patients with severe H1N1 pneumonia and acute respiratory failure. Crit Care Med. 2014 Feb;42(2):313-21. [CrossRef] [PubMed] 